\title{
Manejo de la hiperplasia endometrial con acetato de medroxiprogesterona de depósito
}

\author{
Dres.: Bernardo Moreno Escallón *; Alberto Angulo Roa **; \\ Luis Fernando Botero Arismendi ***; Paula Restrepo de Stiefken ****
}

RESUMEN

Un total de 41 pacientes con diagnóstico histológico de Hiperplasia Endometrial, fueron sometidas a tratamiento médico con Acetato de Medroxiprogesterona de Depósito (AMPD). Un grupo de 26 pacientes (Grupo I), recibió una dosis de $2.5 \mathrm{mgr} / \mathrm{Kgr}$ peso/semana de AMPD durante 8 semanas, y otro grupo de 15 pacientes (Grupo II), recibió una dosis de $5 \mathrm{mgr} / \mathrm{Kgr}$ peso/semana de AMPD durante el mismo tiempo.

Se hizo un seguimiento clínico-histológico, que varió de 6 a 54 meses, con un promedio de 18 meses. Se observó mejoría histológica al mes de iniciada la terapia en el $63 \%$ de los pacientes, y un mes después de terminada la misma, en $100 \%$ de los pacientes de ambos grupos no existía indicios del diagnóstico inicial.

A través del seguimiento se observó mejoría clínica en el $61 \%$ de los pacientes del Grupo I, (16/26), y en el $87 \%$ del Grupo II (13/15). Hubo 10 casos (38\%) de recurrencia clínica en el Grupo I y 3 casos (20\%) en el Grupo II. Solo hubo 1 caso de recurrencia histológica en el Grupo I y ninguna en el grupo II. Aquellos pacientes que presentaron recurrencia, recibieron un segundo ciclo de tratamiento con el doble de la dosis inicial, y de estos 3 casos se consideraron fracasos por evolución clínica desfavorable; sin embargo, al ser intervenidos quirúrgicamente se encontró una causa orgánica (adenomiosis miomatosis), posiblemente responsable de la evolución clínica de estos casos.

Finalmente en forma paralela se verificó la confiabilidad de la Biopsia de Endometrio para el seguimiento de estos casos, encontrándose una excelente correlación entre los resultados histopatológi (Coeficiente de Correlación, $r=0,870 \mathrm{p} « 0.001$ ). El manejo médico de la Hiperplasia ofrece ventajas para aquellas pacientes de riesgo quirúrgico alto, o para quienes no acepten esta via.

\section{SUMMARY}

A total of 41 patients with Endometrial Hyperplasia histological diagnosis were medically treated with Medroxyprogesterone Acetate depot (MPAD). Another group of 26 patients (Group I) received a 2.5 $\mathrm{mgr} / \mathrm{kgr}$ weight/week of MPAD doses during eight (8) weeks and other group of 15 patients (Group II) received a doses of $5 \mathrm{mgr} / \mathrm{kgr}$ weight/week of MPAD during the same period.

It was done a clinical-histological follow-up which varied from 6 to 54 months with an average of 18 months. It was observed histological improvement in $63 \%$ of patients during the first month of teraphy and other month after finished the same in $100 \%$ of patients pertaining to both groups it did not exist any evidence of the initial diagnostic.

During the follow-up it was observed clinical improvement in the $61 \%$ of Group I patients (16/26) and in the $87 \%$ of Group II (13/15). There were ten (10) cases $(20 \%)$ in Grupo II. There was only one case of histological recurrence in group I and none in Group II. Those patients that presented recurrence received a second cycle of treatment with the double of the initial doses and three cases were considered failures due to unfavorable clinical evolution, however, when they were surgically submitted an organic cause was found (adenomyosis, myomatosis) that posibly could be responsible for the clinical evolution in these cases.

Finally, in a parallel way it was verified the Endometrium Biopsy reliability to follow up these cases finding an excellent correlation between the histo-patologic results founded in an uterine curetage and in the Endometrium Biopsy performed to the same patient (Correlation Factor $r=0.870, p 0.001$ ). The medical management of the Endometrial Hyperplasia offers advantages for those patients of quirurical high risk or who do not want to start this way. 


\section{INTRODUCCION}

La hiperplasia endometrial está usualmente asociada con anovulación. El significado de este cambio en el endometrio está relacionado con la edad de la paciente. En la joven con hiperplasia endometrial quien ha menstruado cíclicamente, este cambio será más a menudo visto durante un período de anovulación frecuentemente asociado a stress. Igualmente en la mujer joven con algunos desórdenes endocrinos, como el visto en la enfermedad de ovario poliquístico, la hiperplasia endometrial refleja una estimulación a largo plazo y persistente con estrógenos. La hiperplasia en la mujer premenopáusica no es raro observarla en asociación con falla ovárica, anovulación persistente de estrógenos por el ovario. En la mujer postmenopáusica puede estar asociada con un aumento en la conversión endógena de androstenediona a estrona o excepcionalmente por la presencia de un tumor ovárico funcionante. La hiperplasia también puede ser inducida por el uso de estrógenos exógenos. La fuente exógena incluye el uso de estrógenos para combatir la menopausia o con menor frecuencia, otros estados hipoestrogénicos como la disgenesia gonadal $(23,29)$. Según algunos investigadores, la hiperplasia aunque no ha sido un trastorno al que se haya sometido a investigación minuciosa, tiene relación similar, y tal vez más íntima, con la estimulación de estrógenos no antagonizada, o cuando menos antagonizada en forma insuficiente por la progesterona. En los casos en los que no hay anomalía manifiesta de la secuencia estrógeno/progesterona y una parte del endometrio muestra ciclos normales, la hiperplasia depende muy posiblemente de una anormalidad del "órgano terminal", como deficiencia de receptores que permitan a los estrógenos sólos actuar en las glándulas anormales (30).

Durante muchos años la hiperplasia endometrial ha estado ligada con el cáncer de endometrio. Varios

\footnotetext{
* Profesor Asociado Facultad de Medicina Universidad Javeriana. Jefe Unidad de Esterilidad y Endocrinología Femenina Hospital de San Ignacio.

** Profesor Asociado Facultad de Medicina Universidad Javeriana. Jefe de la Unidad de Ginecología del Departamento de Ginecología y Obstetricia del Hospital de San Ignacio.

*** Instructor de la Facultad de Medicina de la Universidad Javeriana, en el Departamento de Ginecología y Obstetricia del Hospital de San Ignacio.

**** Profesor Asociado Facultad de Medicina Universidad Javeriana en
} el Departamento de Patología del Hospital de San Ignacio.

Trabajo elaborado en el departamento de Ginecología y Obstetricia del Hospital Universitario de San Ignacio, Universidad Javeriana. investigadores $(14,15,42)$ han aportado evidencias bien documentadas sosteniendo que la hiperplasia glanduloquística, adenomatosa, atípica y el carcinoma in situ de endometrio, cuando no son tratadas, pueden evolucionar finalmente a carcinoma invasor.

La mayoría de los oncólogos ginecólogos recomiendan la histerectomía como tratamiento definitivo de la hiperplasia endometrial; sin embargo muchas mujeres presentan problemas médicos (diabetes, enfermedades cardiovasculares o pulmonares severas, nefropatías, obesidad, etc.) que dificultan su manejo quirúrgico. Debido a la falta de criterio unánime para el tratamiento de esta condición, se decidió llevar a cabo el presente estudio con los siguientes objetivos: 1) Establecer un manejo racional y concreto de la Hiperplasia Endometrial glanduloquística y adenomatosa típica. 2) Ofrecer una alternativa de tratamiento a aquellas pacientes con esta patología y con alto riesgo quirúrgico. 3) Ofrecer otra alternativa a aquellas pacientes sin patología orgánica sobreagregada, que no deseen tratamiento quirúrgico. 4) Estudiar la evolución histológica de la Hiperplasia Endometrial, bajo tratamiento con progestágenos. 5) Abrir un punto de partida de investigación de esta patología, para otras investigaciones similares en el futuro.

\section{MATERIAL Y METODOS}

Las pacientes fueron seleccionadas de acuerdo con los siguientes criterios: 1) Pacientes con Hiperplasia Endometrial Quística y Adenomatosa en muestra de Legrado Uterino. 2) Pacientes mayores de 35 años sin deseo de reproducción. 3) Deseo voluntario por parte de la paciente sobre médico. 4) Pacientes con Hiperplasia y riesgo quirúrgico alto.

Por otra parte, fueron excluidas del estudio aquellas pacientes con Hiperplasia Endometrial y otra patología uterina, cervical o anexial sobreagregada; además pacientes que no fuera posible controlar adecuadamente, que no desearan entrar en amenorrea como consecuencia de la terapia, que presentaran contraindicación al uso de progestágenos o que hubiesen recibido terapia hormonal previa.

La clasificación anatomo-patológica de la Hiperplasia Endometrial se hizo con base en la propuesta por la Organización Mundial de la Salud, la cual clasifica la Hiperplasia en: a) Quística; b) Adenomatosa; y c) Atípica (37).

Las pacientes seleccionadas y que ingresaron voluntariamente al estudio, se les practicó examen físico completo, citología vaginal, C.H. y Glicemia pre y post-prandial, y fueron divididas al azar en 2 grupos 
de tratamiento: Un grupo (Grupo I) recibió 150 mgrs. I.M./semana ( $2.5 \mathrm{mgr} / \mathrm{kgr}$. peso/sem.) durante 8 semanas de Acetato de Medroxiprogesterona de Depósito (AMPD), y el otro grupo (Grupo II), recibió 300 mgrs. I.M./semana (5 mgrs/Kgr. peso/sem.) de AMPD durante el mismo tiempo.

Se llevó a cabo un seguimiento clínico mediante control de Peso, Tensión Arterial (T.A.), glicemia y anotación de efectos secundarios, y un seguimiento histológico con Biopsias de Endometrio practicadas al mes, $3,6,9,12$ y 18 meses de iniciada la terapia y luego cada año hasta el momento en el cual fueron tabulados los resultados del estudio.

Se definieron los siguientes términos en el estudio:

Mejoría: Cuando después de finalizado el tratamiento no hubiera sangrado uterino importante (Mejoría clínica), ni Hiperplasia Endometrial (Mejoría Histológica).

Recurrencia: Cuando hubiese persistencia de un sangrado uterino importante o progresión de la $\mathrm{Hi}$ perplasia Endometrial (En los casos de Recurrencia, se administró doble dosis de AMPD, respecto a la dosis que había recibido el paciente inicialmente, y durante 8 semanas).

Falla: Cuando después de un $2^{\circ}$ ciclo de tratamiento (doble de la dosis inicial) en aquellos casos de Recurrencia, no se presentara ni Mejoría clínica ni Mejoría histológica. (Aquellos casos clasificados como "Falla", fueron sometidos a Histerectomía).

En forma paralela se tomó otro grupo de pacientes (en el cual estaban incluidos los pacientes del estudio), que fueron sometidos a Legrado Uterino por Hemorragia Uterina anormal de origen ginecológico, y durante este procedimiento se practicó primero Biopsia de Endometrio con Cureta de Novak (paredes anterior, posterior y laterales del fondo uterino), y esta muestra se colocó en un frasco aparte, y luego se practicó Legrado Uterino con la Cureta de Sims de toda la cavidad, y esta muestra se colocó en otro frasco diferente al primero. Los frascos fueron remitidos al patólogo, con el nombre del paciente, y sin mencionar el origen de la muestra, para comparar los resultados histológicos de las 2 muestras y verificar así la confiabilidad de la Biopsia de Endometrio, como instrumento de seguimiento para este tipo de pacientes.

El análisis estadístico se hizo mediante la aplicación de las pruebas de Chi cuadrado, Test de "student" pareado y no pareado, test de Fischer, regresión linear y Coeficiente de Correlación (r).

\section{RESULTADOS}

De enero $1 / 83$ a Junio 30/87 ingresaron al estudio un total de 54 pacientes con diagnóstico de Hiperplasia Endometrial, de los cuales 13 fueron excluidas por haber presentado intolerancia al AMPD, o porque voluntariamente decidieron retirarse del estudio. De las 41 pacientes restantes, 26 quedaron en el Grupo I de tratamiento y 15 en el Grupo II. El seguimiento clínico histológico varió de 3 a 54 meses, con un promedio de 18 meses. Las características de los 2 grupos de tratamiento están descritos en la Tabla 1. Solamente hubo diferencia estadística significante en la dosis de Acetato de Medroxiprogesterona administrada a cada Grupo y expresada en $\mathrm{mgrs} / \mathrm{Kgr}$ de peso $(\mathrm{p} \ll 0.001)$.

TABLA 1

\section{CARACTERISTICAS DE LOS GRUPOS DE TRATAMIENTO}

\begin{tabular}{|l|c|c|c|}
\cline { 2 - 4 } \multicolumn{1}{c|}{} & $\begin{array}{c}\text { GRUPO I (150) } \\
\text { N }: 26\end{array}$ & $\begin{array}{c}\text { GRUPO II } \\
\text { N: } 15\end{array}$ & (300) \\
\hline EDAD (ANOS) & $51.57 \pm 7.79$ & $48.86 \pm 9.05$ & N.S. \\
\hline PARIDAD & $5.92 \pm 2.92$ & $5.33 \pm 3.17$ & N.S. \\
\hline PESO (kg) & $61.45 \pm 9.62$ & $61.60 \pm 11.87$ & N.S. \\
\hline GLICE MIA $(\mathrm{mg} \%)$ & $87.7 \pm 19.4$ & $84.9 \pm 9.1$ & N.S \\
\hline T.A. SISTOLI (cm) & $12.3 \pm 1.81$ & $13.2 \pm 0.88$ & N.S \\
\hline T.A.DIASTOL $(\mathrm{cm})$ & $7.9 \pm 1.19$ & $8.2 \pm 0.88$ & N.S. \\
\hline SEGUIMIENTO & $18 \pm 3.7(\mathrm{SE})$ & $15.4 \pm 3.18(\mathrm{SE})$ & N.S. \\
\hline MESES & & $5.0 \pm 0.88$ & 0.001 \\
\hline DOSIS (mg./kg.) & $2.5 \pm 0.44$ & &
\end{tabular}

En la Tabla 2 se observa la distribución histológica de la Hiperplasia Endometrial de acuerdo con los Grupos de Tratamiento. Hubo un total de 31 casos con Hiperplasia Quística y 10 con Hiperplasia Adenomatosa, con una proporción aparentemente mayor de ésta última en el Grupo II, sin embargo, no se encontraron diferencias estadísticamente significantes. En un 39\% (16/41) del total de los casos, se produjo Atrofia Endometrial, sin encontrarse diferencias significantes con relación a los Grupos de Tratamiento.

TABLA 2

\section{HISTOLOGIA vS TRATAMIENTO}

\begin{tabular}{|c|c|c|c|}
\hline $\begin{array}{l}\text { HIPERPLASIA } \\
\text { ENDOMETRIAL }\end{array}$ & $\begin{array}{c}\text { GRUPO I } \\
(150 \mathrm{mg})\end{array}$ & $\begin{array}{c}\text { GRUPO II } \\
(300 \mathrm{mg})\end{array}$ & TOTAL \\
\hline QUISTICA & 22 & 9 & 31 \\
\hline ADENOMATOSA & 4 & 6 & 10 \\
\hline TOTAL & 26 & 15 & 41 \\
\hline
\end{tabular}

\begin{tabular}{|c|c|c|c|}
\hline $\begin{array}{c}\text { ATROFIA } \\
\text { ENDOMETRIAL }\end{array}$ & $10 / 26(38 \%)$ & $6 / 15(40 \%)$ & $16 / 41(39 \%)$ \\
\hline
\end{tabular}


Se encontró una mejoría histológica al mes de iniciada la terapia en el $63 \%$ (26/41) del total de pacientes, con una distribución de un 55\% de mejoría (14/26) en el Grupo I y 75\% (11/15) en el Grupo II, sin existir diferencias significativas. Un mes después de finalizada la terapia, hubo $100 \%$ de mejoría histológica en ambos grupos (Fig. 1). En las fotografías 1 a 4 se ilustra la evolución histológica con el tratamiento, de 4 pacientes con Hiperplasia Quística y Adenomatosa respectivamente (pacientes L.O., M.M., I.N. y V.M.)

FIGURA I

\section{EVOLUCION HISTOLOGICA HIPERPLASIA ENDOMETRIAL (41 CASOS)}

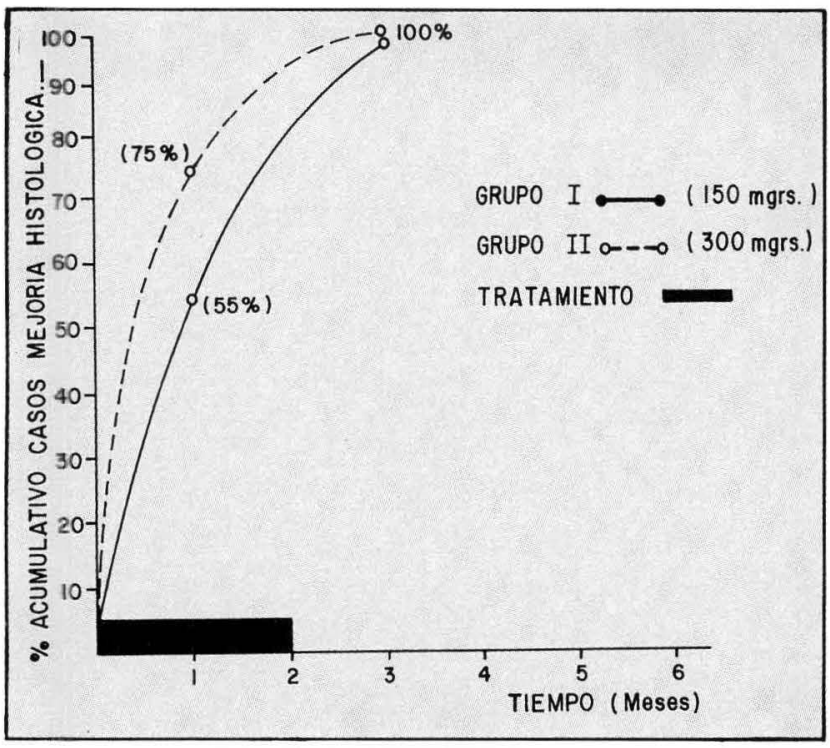

Con respecto a la evolución clínica, se observó mejoría clínica en el $61 \%$ de los pacientes del Grupo I (16/26) y en $87 \%$ del Grupo II (13/15), diferencias no estadísticamente significantes. Hubo 10 casos (38\%) de recurrencia clínica en Grupo I y 3 casos en el Grupo II (20\%). Sólo se presentó I caso de recurrencia histológica en el Grupo II (Fig. 2) y hasta el momento en el que se escribió este manuscrito (Mayo/90) no había sido reportada ninguna otra recurrencia histológica.

Aquellos pacientes que presentaron recurrencia, recibieron un segundo ciclo de tratamiento con el doble de la dosis inicial y de estos 3 casos se consideraron fracasos o fallas por evolución clínica desfavorable (Fig. 2). Estos pacientes fueron intervenidos quirúrgicamente; sin embargo, el estudio anatomopatológico de la pieza quirúrgica, mostró causas orgánicas (adenomiosis, miomatosis), posiblemente responsables de la evolución clínica de estas pacientes. En el estudio del endometrio de estos úteros no se encontró Hiperplasia Endometrial.

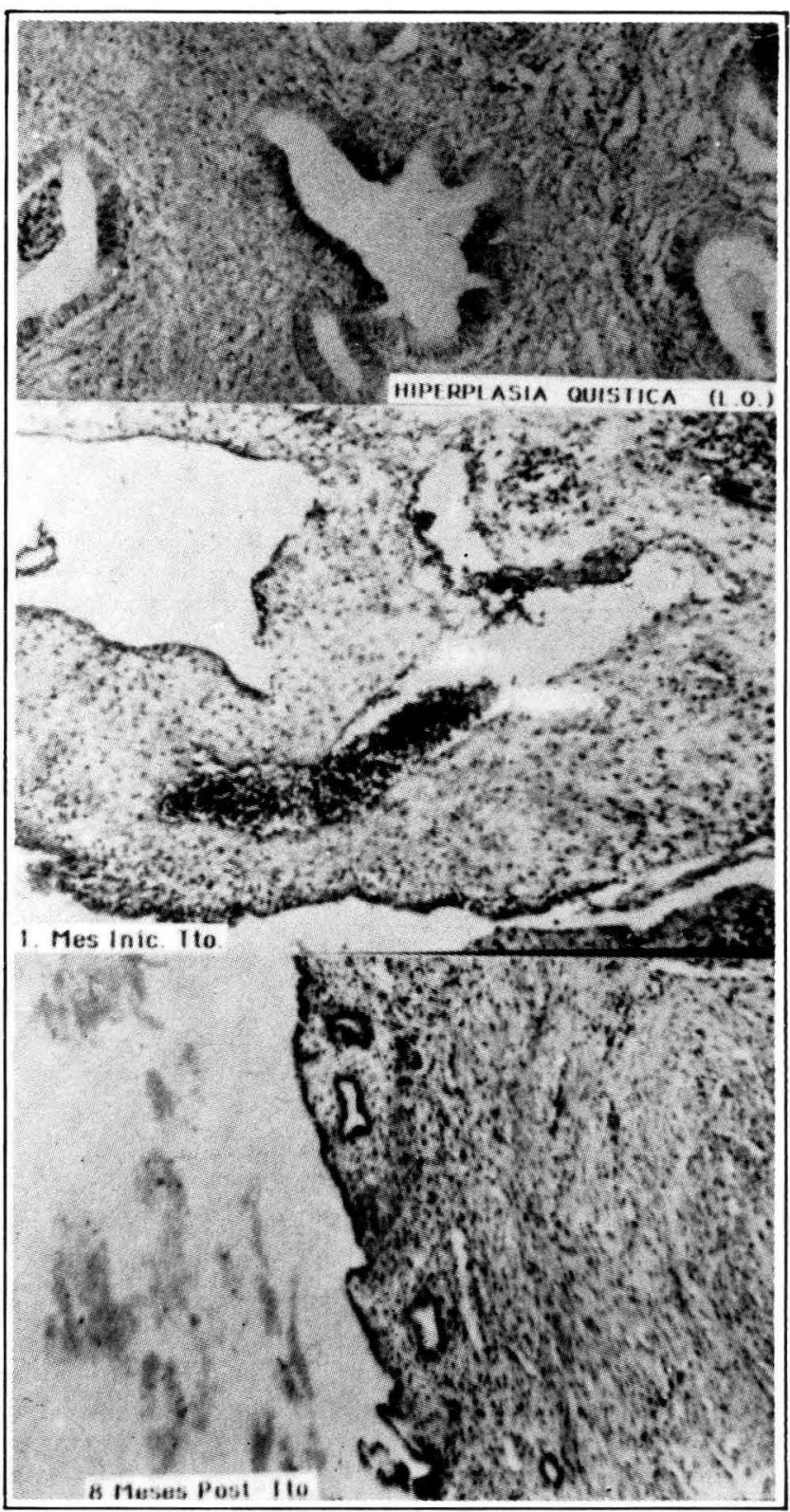

Se hizo un análisis del grupo total de las 14 pacientes que presentaron Recurrencia, teniendo en cuenta la suma de los Grupos I y II se comparó con el grupo restante de 27 pacientes que no presentó Recurrencia. Solamente hubo una ligera pero significante diferencia estadística en la dosis/Kgr. de peso de AMPD entre el Grupo Recurrente y el Grupo de Mejoría (p«0.05); el resto de características fueron similares (Tabla 3). Tampoco se encontraron diferencias comparables en el Grupo Recurrente cuando se analizó el número de casos de Hiperplasia Quística y Adenomatosa con relación a los Grupos I y II de tratamiento (Tabla 4). 


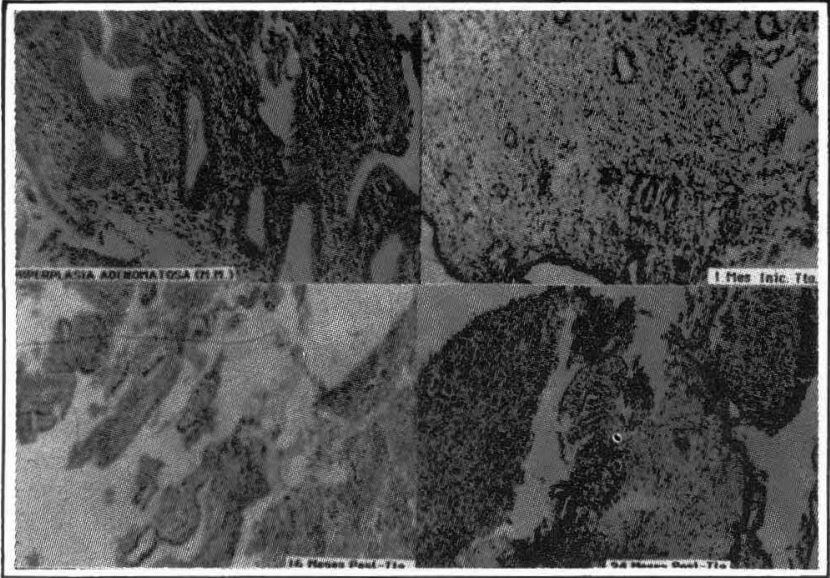

FOTO 3

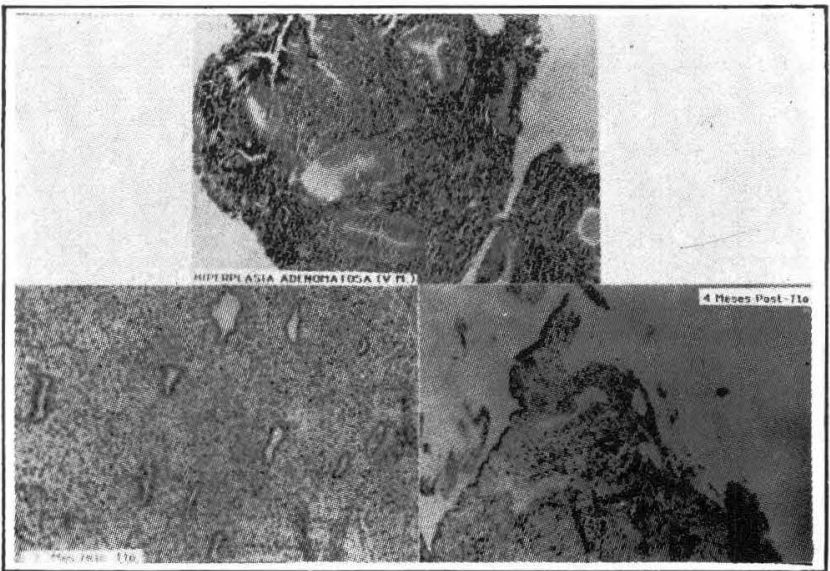

FIGURA 2

EVOLUCION CLINICA HIPERPLASIA ENDOMETRIAL (41 CASOS)

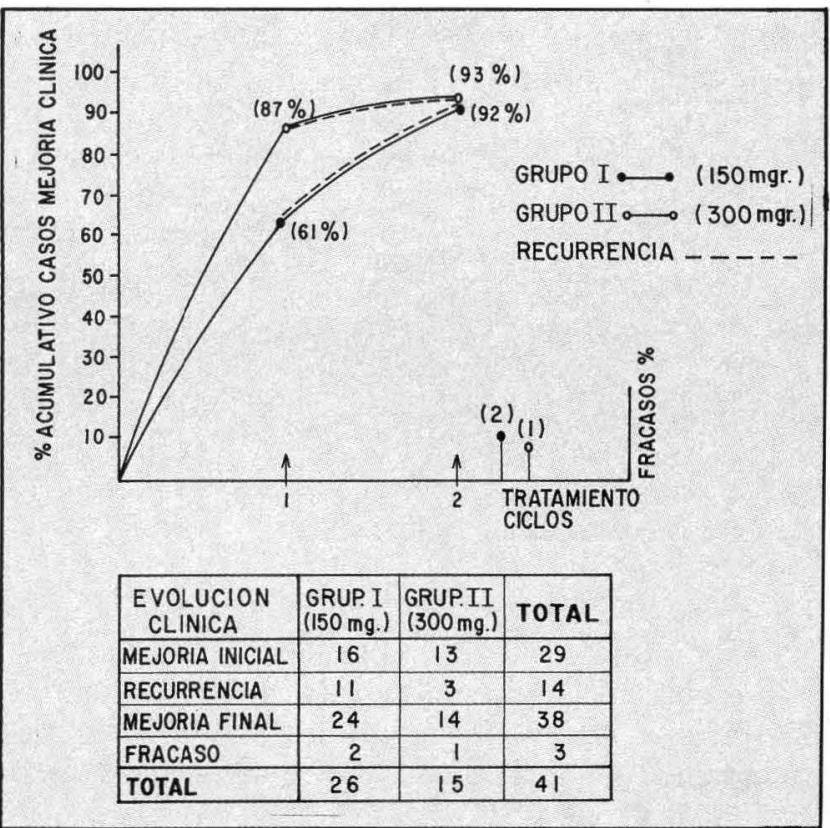

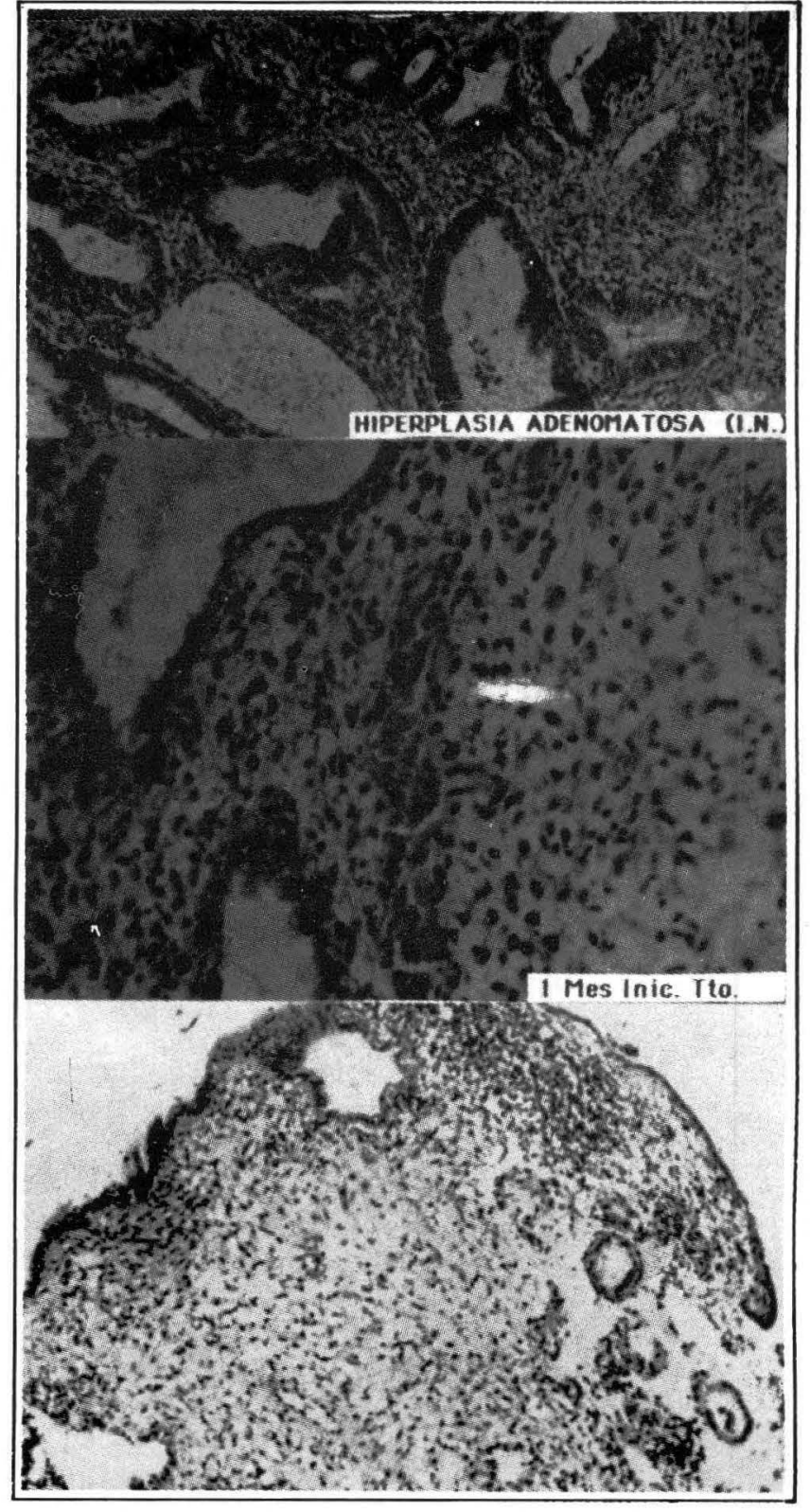

En la Figura 3, se aprecia la distribución a través del tiempo de los casos que presentaron Recurrencia clínica después de terminado el tratamiento. Aunque el número de casos no se presta para un análisis estadístico, sin embargo, se observa una tendencia a disminuir el número de casos después de 7 meses de finalizado el tratamiento. Sólo hubo 1 caso de recurrencia histológica a los 11 meses de terminado el tratamiento.

Finalmente, se encontró una excelente correlación $(r=0.870 ; \mathrm{p} \ll 0.0001)$ entre los resultados anatomopatológicos encontrados en la Biopsia de Endometrio $y$ el Légrado Uterino practicados a un mismo paciente (Fig. 4). 
TABLA 3

CARACTERISTICAS DEL GRUPO RECURRENTE

\begin{tabular}{|l|c|c|c|}
\cline { 2 - 4 } \multicolumn{1}{c|}{} & $\begin{array}{c}\text { RECURRENCIA } \\
\text { N }: 14\end{array}$ & $\begin{array}{c}\text { MEJORIA CLIN. } \\
\text { N: } 27\end{array}$ & $P$ \\
\hline EDAD (AÑOS) & $50.0 \pm 5.75$ & $50.8 \pm 9.4$ & N.S. \\
\hline PARIDAD & $6.46 \pm 2.00$ & $5.44 \pm 3.35$ & N.S. \\
\hline PESO (Kgs.) & $62.35 \pm 13.14$ & $59.74 \pm 9.46$ & N.S. \\
\hline GLICEMIA (mgs.\%) & $92.1 \pm 23.3$ & $81.3 \pm 11.9$ & N.S. \\
\hline T.A. SISTOL./cm) & $12.6 \pm 1.7$ & $12.6 \pm 1.61$ & N.S. \\
\hline T.A. DIASTO.(cm.) & $8.08 \pm 0.9$ & $7.94 \pm 1.25$ & N.S. \\
\hline H.ADENOMATOS. & 2 & 8 & N.S. \\
\hline H.QUISTICA & 12 & 19 & N.S. \\
\hline $\begin{array}{l}\text { SEGUIMIENTO POST } \\
2^{\circ} \text { CICLO }\end{array}$ & $12 \pm 2.8$ (S.E.) & - & - \\
\hline $\begin{array}{l}\text { TIEMPO ENTRE TTO } \\
\text { INICIAL Y RECURREN. }\end{array}$ & $6.9 \pm 2.2$ (S.E.) & - & - \\
\hline DOSIS (mg./Kg) & $2.87 \pm 1.1$ & $3.71 \pm 1.4$ & $<0.05$ \\
\hline
\end{tabular}

TABLA 4

PATOLOGIA EN LOS CASOS RECURRENTES

\begin{tabular}{|c|c|c|c|}
\hline $\begin{array}{c}\text { HIPERPLASIA } \\
\text { ENDOMETRIAL }\end{array}$ & $\begin{array}{c}\text { GRUPO I } \\
(150 \mathrm{mg})\end{array}$ & $\begin{array}{c}\text { GRUPO II } \\
(300 \mathrm{mg})\end{array}$ & TOTAL \\
\hline QUISTICA & 9 & 3 & 12 \\
\hline ADENOMATOSA & 2 & 0 & 2 \\
\hline TOTAL & 11 & 3 & 14 \\
\hline
\end{tabular}

FIGURA 3

TIEMPO ENTRE TRATAMIENTO Y

RECURRENCIA (6.8 \pm 5.2 Meses)

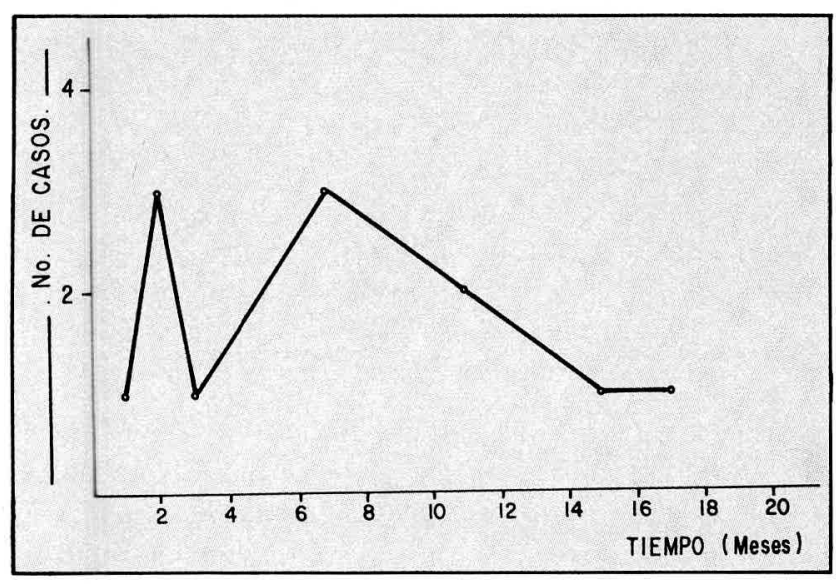

DISCUSION

El uso de los progestágenos en el manejo de la Hiperplasia Endometrial se inició hace un poco más de 30 años. A pesar de todo este tiempo, no existe un número proporcional de publicaciones a éste respecto. El mayor número de reportes en la literatura médica con relación a su uso, está enfocado al tratamiento del carcinoma de endometrio. En el año 1951, Kelly (21)

\section{FIGURA 4 \\ CORRELACION LEGRADO UTERINO Y BIOPSIA ENDOMETRIAL (76 CASOS)}

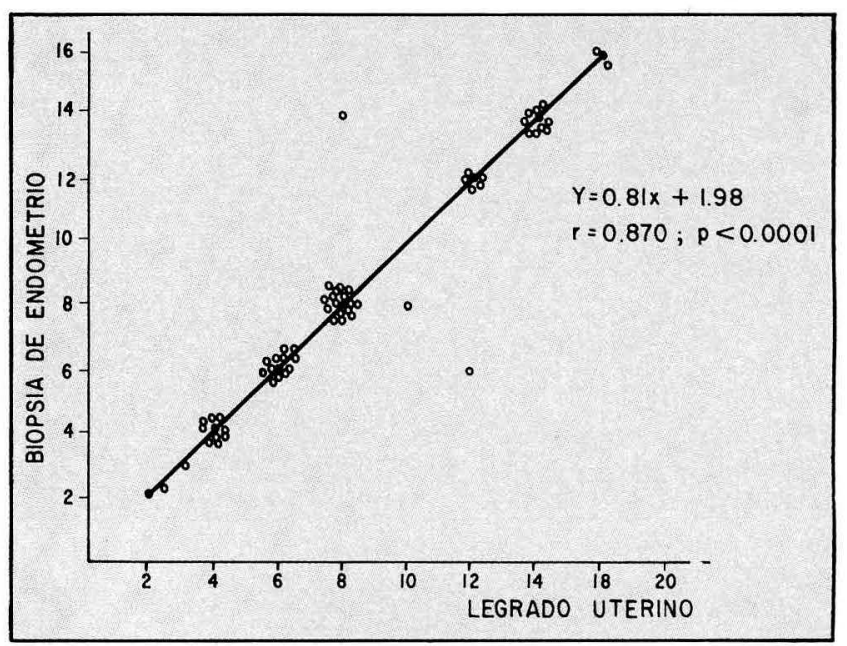

encontró que la terapia con progesterona era efectiva en el tratamiento del carcinoma de endometrio primario y metastásico. En 1959, Kitsner (22) reportó que los progestágenos tenían un efecto beneficioso sobre la hiperplasia del endometrio. Wentz, Eichner y Podratz (7, $27,44)$ han confirmado la efectividad de varios tipos de progestágenos en el manejo de la hiperplasia endometrial. En 1953, Wellenback y Rakoff (43) demostraron en hamsters castrados, que la hiperplasia endometrial inducida mostraba rápida regresión cuando se administraba progesterona. En el año 56, aparecieron agentes progestacionales sintéticos que pudieron utilizarse en la investigación clínica. Las publicaciones de Kelley y Baker (20) despertaron interés al describir los efectos del caproato de 17 alfa-hidroxiprogesterona en el cáncer endometrial metastásico. Gal y col. en 1983 trataron 52 pacientes mujeres post-menopáusicas con hiperplasia adenomatosa y carcinoma in situ de endometrio utilizando el Megestrol durante un período de 9 a 104 meses y obteniendo una remisión de la lesiones endometriales en el $90 \%$ de los casos (11).

El tratamiento de la Hiperplasia Endometrial no debe ser considerado solamente como el control de unos cambios anatomopatológicos en sí, sino de tratar de controlar los efectos etiológicos y epidemiológicos extrauterinos que contribuyen a iniciar y perpetuar un proceso, que sino se trata, puede llevar al paciente a estados invasivos de la enfermedad.

Por esta razón si se decide hacer un manejo médico y un largo seguimiento de las pacientes, posiblemente un control de la enfermedad a nivel local "Histerectomía Médica" y un control de los factores extrauterinos conduzca a unos mejores resultados. Esto lógicamente conlleva implicaciones en el cambio de hábito de las pacien- 
tes con respecto al cigarrillo, ejercicio, control de peso, y esto obviamente, no lo puede lograr solamente la droga elegida.

Varios tipos de progestágenos se han utilizado en el manejo de la Hiperplasia Endometrial, como el megestrol, dimestisterona, capronato de 17 hidroxiprogesterona, acetato de medroxigesterona, observándose regresión de la Hiperplasia Endometrial dentro de los tres meses de iniciada la terapia (11), tiempo comparable con el obtenido en el presente estudio.

El problema ha surgido en la forma de seguimiento, tratamiento a largo plazo y un control unificado de estas pacientes. Por esta razón muchos ginecólogos recomiendan Histerectomía a estas pacientes, la cual no siempre puede ser llevada a cabo, debido a que existen contraindicaciones como la obesidad, diabetes, enfermedades cardiovasculares o pulmonares severas, o simplemente la paciente no desea en primera instancia ser operada.

Quienes recomiendan la Histerectomía, deben tener en cuenta los riesgos vs. los beneficios, ya que este procedimiento no deja de tener morbilidad y las cifras de mortalidad entre 4.1 y 85.1 x 10.000 histerectomías (45) deben contar para tomar esta decisión. Por otra parte, los costos y posibilidades económicas del paciente, también deben ir a uno de los platillos de la balanza.

Conociendo los resultados de un tratamiento médico, se le debe ofrecer esta oportunidad al paciente y dejar el manejo quirúrgico como última alternativa, si la primera fracasa.

La administración de progesterona reduce el contenido de receptores para estrógenos en el útero de la rata (18). Otros autores han demostrado un efecto similar en el endometrio humano (40). Este efecto ocurre concomitantemente con la inducción de la enzima 17 Beta-estradiol dehidrogenasa, enzima que cataboliza el estradiol, efecto debido a los progestágenos. Además de reducir el contenido de receptores estrogénicos, bloquea la nueva síntesis de estos receptores o reciclaje (5).

El acetato medroxiprogesterona de depósito, derivado sintético de la progesterona tiene los mecanismos de acción mencionados anteriormente y además bloquea el pico de LH y en altas dosis produce supresión de las suprarrenales (26), e incrementa la rata de depuración de la testosterona y disminuye su producción (19).

Aunque en el presente estudio no se determinaron las gonadotrofinas séricas ni esteroides; sin embargo, estas acciones del AMPD, podrían considerarse como una ventaja en el manejo inicial de la Hiperplasia Endo- metrial, si se tiene en cuenta que en la mujer post-menopáusica la secreción ovárica de androstenediona y testosterona continúa y es estimulada por el nivel elevado de gonadotrofinas propio de las menopáusicas, además las adrenales contribuyen con el $85 \%$ de la secreción de androstenediona la cual es convertida a estrona en el tejido graso $(1,13)$.

Tampoco fueron determinados el colesterol ni las lipoproteínas de alta y baja densidad; sin embargo, a este respecto el AMPD podría tener también ventajas sobre otros progestágenos sintéticos como lo han demostrado los estudios de Hirvonen en 1981 (16), Mattsson (25) y Silferstolpe (33), comparando el efecto de varios progestágenos sobre la disminución de la fracción de colesterol ligada a las lipoproteínas de alta densidad, sobre la cual el acetato de medroxiprogesterona no mostró efecto (28). Claro está que utilizando dosis más altas de AMPD, como 150 a $1000 \mathrm{mgr} / \mathrm{semana}$ durante 6 meses es posible alterar el metabolismo de las lipoproteínas de alta densidad $(6,9)$, sin embargo, dosis más bajas a estas se utilizaron en el presente estudio.

El análisis de los resultados muestra que la única diferencia estadísticamente significante entre el Grupo I y el Grupo II fue la dosis de AMPD/Kgr de peso (Tabla 1), a pesar de haber ingresado los pacientes en forma indiscriminada a uno u otro grupo; esto hace que en términos de resultados, sean comparables ambos grupos.

Al parecer existe una respuesta individual de cada paciente al tratamiento de la hiperplasia, ya que no se encontraron diferencias cuando se compararon ambos grupos, con respecto al tipo de Hiperplasia, y en igual forma ésto está soportado por el hecho que el porcentaje de Atrofia Endometrial que se produjo en cada uno de los grupos, fue muy similar (Tabla 2). Algo parecido se observó cuando en el Grupo Recurrente, se compararon los grupos de tratamiento versus los tipos histológicos (Tabla 4). Aunque el grupo fue pequeño, sin embargo, la recurrencia al parecer no depende de un tipo histológico determinado, sino de una sensibilidad individual. Por otra parte existe una ligera tendencia a producirse una recurrencia clínica, la cual es dependiente de la dosis de AMPD (Tabla 3).

Se ha descrito un aumento de peso con el uso de AMPD desde 1.4 libras hasta 9 libras durante el primer año de uso $(4,24,32)$. En el presente estudio hubo diferentes respuestas; como aumentos de peso desde 0.4 hasta 5.5 kilos, así como disminución del mismo desde 0.5 a 1.2 kilos, sin embargo, al comparar el peso total antes del tratamiento con el peso 1, 3, 6, 9, 12 y 18 meses después, no se encontraron diferencias estadísticas. 
Aunque algunos investigadores han mostrado que la AMPD aumenta las concentraciones de glucosa en sangre $(3,12,35)$, no existe un acuerdo general a este respecto $(31,38,39,41)$. El análisis de este aspecto no mostró diferencias significantes en las glicemias basal y post-prandial antes y después del tratamiento.

Tampoco se encontraron diferencias en las presiones sistólicas y diastólicas, antes y después del tratamiento; aunque algunos autores han encontrado aumentos en la presión diastólica, la mayoría de reportes de la literatura son contradictorios (24).

Por más de 50 años la dilatación cervical y el curetaje han sido aceptados como procedimientos diagnósticos para obtener muestras adecuadas para diagnóstico de lesiones endometriales, sin embargo, este procedimiento sigue siendo más costoso, más riesgoso, conlleva más stress para el paciente y a veces se obtiene un bajo número relativo de diagnósticos de lesiones endometriales graves. Cuando se compara con otros procedimientos más simples de uso ambulatorio y a nivel del consultorio, no ofrece mayores ventajas reales. Recientemente, Smith y col. hacen un llamado a los investigadores sobre la necesidad de revisar la práctica del curetaje en todos los casos de Hemorragia Uterina Anormal (34). Un sin número de estudios han sido llevados a cabo evaluando diferentes formas más sencillas de obtener una muestra endometrial, como el lavado, aspiración, cepillado, biopsia etc. $(2,8,17,36)$, sin embargo, el mayor problema que existe en el diseño de estos reportes, es que el instrumento de diagnóstico utilizado, no fue seguido de curetaje o histerectomía, para poder comparar real y objetivamente la confiabilidad del método.

En el presente estudio, se llevó a cabo una evaluación de la Biopsia de Endometrio en pacientes con sangrado uterino anormal y se comparó con el Curetaje Uterino practicado a un mismo paciente, y los resultados de anatomía patológica de las muestras tomadas por ambos métodos, mostraron una alta correlación, lo que significa, que la Biopsia de endometrio en nuestras manos, tiene una excelente confiabilidad, y que las pacientes del estudio, pueden ser debidamente controladas con este instrumento. Este resultado puede ser esperado, si se compara con algunos reportes sobre la biopsia de endometrio, que muestran un índice de precisión para el diagnóstico del carcinoma de endometrio por encima del 91\% $(10,17)$. Por esta razón creemos que esta pequeña experiencia, utilizando un diseño comparando la biopsia de endometrio y el curetaje en un mismo paciente, podría ponerse en práctica en todo paciente que vaya a ser sometido a curetaje por hemorragia uterina anormal, con el objeto de poder responder en el futuro sobre la justificación de este último procedimiento y sólo ponerlo en práctica en los casos especiales.

\section{CONCLUSIONES}

1. El acetato de Medroxiprogesterona (AMP), debe continuar siendo considerado, como una herramienta terapéutica útil, en el manejo de la Hiperplasia Endometrial.

2. Todo paciente con Hiperplasia Endometrial y sangrado uterino de causa no orgánica, se le debe ofrecer en primera instancia, un tratamiento médico con AMP antes de tomar una alternativa quirúrgica.

3. La Biopsia Endometrial efectuada adecuadamente, es un instrumento útil y confiable para el seguimiento histológico de la enfermedad.

4. Se recomienda practicar biopsias endometriales de seguimiento, 6 y 12 meses de finalizada la terapia. y luego en forma anual.

5. De acuerdo con los resultados del presente trabajo. se recomienda una dosis de $5 \mathrm{mgr} / \mathrm{Kgr}$. de peso/semana: 8 semanas, que es la Media - 1 DS de la dosis administrada al grupo que no presentó recurrencia.

6. Se recomienda para nuevas pacientes y estudios futuros, poner en práctica métodos paraclínicos, que contribuyan a descartar una causa orgánica de la hemorragia uterina, y acelerar así el manejo definitivo del paciente.

\section{BIBLIOGRAFIA}

1. ABRAHAN, G. Ovarian and adrenal contribution to peripheral androgens during the menstrual cycle $\mathrm{J}$ Clin Endocrinol Metab 39: 340, 1974.

2. BIBBO, M. Accurancy of three sampling technics for the diagnosis of endometrial cancer and hyperplasia $J$ Reprod Med 27: 622, 1982.

3. CAMANNI, F.; MASSARA, F.; MOLINATTI, G.M. The cortisone like effect of -alfa-methyl-17 alfaacetoxiprogesterone in the adrenalectomized man. Acta Endocrinol 43: 477-483, 1963.

4. CHINNATAMBY, S.A. Comparation if the long-acting contraceptive agents norethisterone oenan thate and medroxyprogesterone acetate. Australian and New Zealand J Obstet Gynecol 11: 123-236, 1971.

5. CLARK, J.K.; ANDERSON, J.N.; PECK, E.J. Estrogen receptor anti-estrogen complex: Atypical binding by uterine nuclei and effects on uterine growth, Steroids 22: 707, 1974.

6. CRONA, N.; ENK, L.; SAMSIOE, G.; SILFVERSTOLPE, G.; SKRYTEN, A. High-dose depot-medroxyprogesterone acetate (DMPA)- effects on lipid and lipoprotein metabolism. Eur. J Obstet Gynecol Reprod Biol. 16: 97 , 1983. 
7. EICHNER, E.; ABELLERA, M. Endometrial hyperplasia treated by progestins. Obstet Gynecol 38: 739, 1971.

8. EINERTH, Y. Vacuum curettage by the vabra method. Acta Obstet Gynecol 61: 373, 1982.

9. FAHRAEUS, L.; SYDSJOE, A.; WALLENTIN, L. Lipoprotein changes during treatment of pelvic endometriosis with medroxiprogesterone acetate. Fertil Steril 45: 503, 1986.

10. FERENCZY, A.; SHORE, GURALNICK, M.; GELFAND, M.M. The Kevorkian curette:an appraisal of its endometrial evaluation. Obstet Gynecol 54:262, 1979.

11. GAL, D.; EDMAN, C.D.; VELLIOS, F.; FORNEY, P. Longterm effect of megestrol acetate in the treatment of endometrial hyperplasia Am J Obstet Gynecol 146: 316 , 1983.

12. GERSHBERG, H.; ZORRILLA, E.; HERNANDEZ, A.; HULSE, M. Effects of medroxyprogesterone acetate on serum insulin and growth hormone levels in diabetics and potetial diabetics. Obstet Gynecol 33: 383-389, 1969.

13. GREENBLATT, R.B.; COLLE, M.L.; MAHESH, V.B. Ovarian and adrenal steroid production in the post menopausal ovary. Obstet Gynecol 47: 383, 1976.

14. GUSBERG, S.B. Precursor corpus carcinoma: Estrogens and adenomatous hyperplasia Am J Obstet Gynecol 54: $905,1947$.

15. HERTIGAT, A.T., Sommers Sc. Genesis of endometrial cancer. I Study of prior biopsies. Cancer 2: 296, 1949.

16. HIRVONEN, E.; MALKONEN, M.; MANNINEN, V. Effects of different progestogens on lipoproteins during postmenopausal therapy. New Eng J Med 304: 560, 1981.

17. HOFMEISTER, F.J. Endometrrial biopsy: another look Am J Obstet Gynecol 118: 773, 1974.

18. HSUEH, A.J.W.; PECK, E.J.; CLARK, J.H. Progesterone antagonism of the estrogen receptor and estrogen induced uterine growth. Nature 254: 337, 1975.

19. JUDD, K.L.; LUCAS, W.E.; YEN, S.S.C. 'Effect of oophorectomy on circulating testosterone and androstenedione levels in patients with endometrial cancer Am J Gynecol 118: 793, 1974.

20. KELLEY, R.W.; BAKER, W.H. Effects of 17 -alphahydroxyprogesterone caproate on metastasic endometrial cancer. Conference on Experimental Clinical Cancer Chemotherapy, Monograph 9 National Cancer Institute, Bethesda, 1960: 235.

21. KELLY, R.M. In proceeding of the Second Conference of Steroids and Cancer. Council of Pharmacy and Chemistry American Medical Association, Chicago 116 1951.

22. KISTNER, R.W. Histological effects of progestins on hyperplasia and carcinoma in situ of the endometrium Cancer 12: 1106, 1959.

23. KISTNER, R.W. HAMMOND, CH. B., KAUFAM, R.H. Menopause course. Sylabus of the American Fertility Society XI Annual postgraduate course, Houston Texas March 19: 7, 1980.

24. LEIMAN, G. Depo-medroxyprogesterone acetate as a contraceptive agent: its effect on weight and blood pressure Am J Obstet Gynecol 144(1): 97-102, 1972.

25. MATTSSON, L.; CULLBERG, SAMSIOE, G. Influence of esterified estrogens and medroxyprogesterone on lipid metabolism and sex steroids. A study in oophorectomized women. Hormone Metab Res 14: 602, 1982.

26. PANNUTI, F.; MARTONI, A. High dose of medroxiprogesterone acetate in oncology. History clinical and parmacokonetics. In: Cavalli F., McGuire WL. Pannuti Robustelli Della Cuna G (Eds): Proceeding of the international Symposium on Medroxyprogesterone acetate. Excerta Medica Geneve Switzerland pp 24-26, 1982.

27. PODRATZ, K.C.; O'BRIEN, P.C.; MALKASIAN, G.D.; DECKER, D.C.; JEFFERIES, J.A.; EDMONSON, J.H. Effects of progestational agents in treatment on Endometrial Carcinoma Obstet Gynecol 66: 106, 1985.

28. RANDALL, B.B.; SUBIR, R.; LOBO, R. Comparision of lipid and androgen levels after conjugated estrogen or Depo-medroxiprogesterone acetate treatment in postmenopausal women. Obstet Gynecol 66:216, 1985.

29. SCHIFF, I.; SELA, H.K.; CRAMER, D.; TULCHINSKY, D.; RYAN, K. Endometrial hyperplasia in women on cyclic or continuous estrogen regimens. Fertil Steril 37:79, 1982.

30. SCULLY, R.E. Definition of endometrial carcinoma precursors. Clin Obstet Gynecol 25: 39, 1982.

31. SCHWALliE, C.; ASSENZO, J.R. Contraceptive useefficacy study utilizing medroxiprogesterone acetate administered as an intramuscular injection once every 90 days. Fertil Steril 24: 331-339, 1973.

32. SEYMOUR, R.J.; POWELL, L.C. Depomedroxyprogesterone acetate as a contraceptive. Obstet Gynecol 36: 589-596, 1970.

33. SILFERSTOLPE, G.; GUSTAFSSON, A.; SAMSIOE, G.; SYANBORG, A. Lipid metabolic studies in oophorectomized: effects on serum lipids and lipoproteins of three synthetic progestogens. Maturitas 4: 103, 1983.

34. SMITH, J.J.; SCHULMAN, H. Current dilatation and curettage practice: A need for revision. Obstet Gynecol 65: 516-518, 1985.

35. SPELLACY, W.N.; MCLEOD, G.W.; BUHI, W.C.; BIRK, S.A.; MCCREARY, S.A. Medroxyprogesterone acetate and carbohydrate metabolism: measurement of glucose, insulin and growth hormone during 6- months'time Fertil Steril 21: 457-463, 1970.

36. SUAREZ, R.A.; GRIMES, D.A.; MAJAMUDAR, B. Diagnostic endometrial aspiration with the Karman cannula J Reprod Med 28: 41, 1983.

37. TAVASSOLI, F.; KRAUSS, F.T. Endometrial lesions in uteri resected for atypical endometrial hyperplasia Am J Clin Pathol 70: 770, 1978.

38. TUTTLE, S.; TUKINGTON, V.E. Effects medroxyprogesterone acetate on carbohydrate metabolism Obstet Gynecol 43: 685-692, 1974.

39. TYKER, E.T.; LEVIN, M.; ELLIOT, J.; DOLMAN, H. Present status of injectable contraceptives: results of seven years study Fertil Steril 21: 469-481, 1970.

40. TSENG, L.; GURPIDE, E. Effect of progestins on estradiol levels in human endometrium $\mathrm{J}$ Clin Endocrinol Metab 41: 402, 1975.

41. VECCHIO, T.J. Injectable medroxyprogesterone acetate contraception: metabolic and endocrine effects. J Reprod Med 10: 193-196, 1973.

42. VELLIOS, F. Endometrial hyperplasia and carcinoma in situ. Gynecol Oncol 2: 152, 1974.

43. WELLENBACK, D.L.; RAKOFF, A.E. Hyperplasia of the endometrium J Albert Einstein Med Center 2: 3, 1953.

44. WENTZ, W.B. Treatment of persistent endometrial hypherplasia with progestins Am J Obstet Gynecol 96: 999, 1966.

45. WINGO, P.A.; HUEZO, C.M.; RUBIN, G.L.; ORY, H.W.; PETERSON, H.B. The mortality risk associated with hysterectomy Am J Obstet Gynecol 152: 803, 1985. 